\title{
INTEGRAL INVARIANT FUNCTIONS ON THE NILPOTENT ELEMENTS OF A SEMISIMPLE LIE ALGEBRA
}

\author{
MICHAEL A. GAUGER
}

\begin{abstract}
Let $L$ be a semisimple Lie algebra over an algebraically closed field of characteristic zero. It is shown that there is a finitely generated ring $\boldsymbol{R}$ of integral invariant functions such that for nilpotent elements $x$ and $y$ of $L$, one has $x$ conjugate to $y$ if and only if $f(x)=f(y)$ for all $f$ in $R$. The result is analogous to Chevalley's determination of conjugacy classes of semisimple elements by the ring of invariant polynomial functions.
\end{abstract}

Introduction. The usefulness of invariant polynomial functions on a semisimple Lie algebra $L$ in determining conjugacy classes of semisimple elements is well known [2, Proposition 10] (conjugacy here is with respect to the adjoint group). The ring of invariant polynomial functions is isomorphic to a polynomial ring in $l$-variables $(l=\operatorname{rank}(L))$, and two semisimple elements $x, y$ in $L$ are conjugate if and only if $f(x)=f(y)$ for each invariant polynomial function $f$ (or each such $f$ in a set generating the ring of invariants). On the other hand, these functions are useless in studying conjugacy classes of nilpotent elements, since each invariant polynomial vanishes at all nilpotent elements. In the language of invariant theory, the nilpotent elements are the "nullforms". We introduce here a finitely-generated ring $\mathbf{R}$ of integral invariant (i.e. constant on conjugacy classes) functions on the subvariety of nilpotent elements of a semisimple Lie algebra, and show that if $x$ and $y$ are nilpotent elements, then they are conjugate if and only if $f(x)=f(y)$ for all $f$ in $\mathbf{R}$. $\mathbf{R}$ is also shown to contain all the obvious integral invariant functions.

The main results. Let $N$ be the subvariety of nilpotent elements of a semisimple Lie algebra $L$ defined over an algebraically closed field of characteristic zero, and let $x$ be in $N$. We write $x_{V}$ for the action of $x$ on any finite-dimensional $L$-module $V$. It is well known that $x_{V}$ is a nilpotent endomorphism of $V$. Set

$$
r_{V, k}(x)=\operatorname{rank}\left(x_{V}\right)^{k}
$$

and notice that $r_{V, k} \equiv 0$ for all $k \geqslant \operatorname{dim} V$. Each such function is invariant

Received by the editors February 22, 1977 and, in revised form, May 18, 1977.

AMS (MOS) subject classifications (1970). Primary 17B20.

Key words and phrases. Semisimple Lie algebra, conjugacy, invariant function, nilpotent and semisimple elements. 
since conjugate elements act similarly on each module, and since similar linear transformations have identical ranks. We let $\mathbf{R}$ be the ring generated (finitely) over $\mathbf{Z}$ by all $r_{V, k}$ where $V$ runs through the fundamental $L$-modules. That $\mathbf{R}$ determines conjugacy classes of nilpotent elements of $L$, follows directly from:

THEOREM 1 (GAUGER [1]). Let $x, y$ be elements of the semisimple Lie algebra L. Then $x$ is conjugate to $y$ if and only if $x_{V}$ is similar to $y_{V}$ for every fundamental L-module $V$.

THEOREM 2 (WEYR [3, P. 73]). Let $x, y$ be nilpotent $n \times n$ matrices over any field. Then $x$ and $y$ are similar if and only if $\operatorname{rank}\left(x^{k}\right)=\operatorname{rank}\left(y^{k}\right)$ for all $k<n$.

We state this property of $\mathbf{R}$ as

THEOREM 3. Let $x$ and $y$ be nilpotent elements in the semisimple Lie algebra $L$. Then $x$ is conjugate to $y$ if and only if $f(x)=f(y)$ for all $f$ in $\mathbf{R}$.

Proof. As the functions in $\mathbf{R}$ have been shown to be invariant, we need only show the sufficiency of the condition. Theorem 2 implies that $x$ and $y$ act similarly on each fundamental $L$-module $V$. Theorem 1 can then be invoked to deduce that $x$ and $y$ are conjugate.

REMARK. In some cases a smaller generating set for $\mathbf{R}$ can be specified. If $L$ is simple of type $A, B, C,(D)$ then one needs only the $r_{V, k}$ where $V$ is the first fundamental (i.e. natural geometric) representation (first fundamental representation or the spin representation). This follows since Theorem 1 requires a small number of fundamental modules when $L$ is classical simple [1]. Some headway is also made in [1] in reducing the requirements of Theorem 1 for the exceptional simple Lie algebras.

Our next aim is to show that $\mathbf{R}$ contains all the obvious integral invariant functions on $N$. The obvious integral invariants one can define for a nilpotent element $x$, are things like the rank, nullity and number of Jordan blocks of a given size, of $x_{V}$ or $\left(x_{V}\right)^{t}$ for some $L$-module $V$. Let $n_{V, k}(x)$ be the number of $k \times k$ Jordan blocks in the Jordan form of $x_{V}$. It is well known, and easily shown by induction, that

$$
n_{V, k}(x)=r_{V, k+2}(x)-2 r_{V, k+1}(x)+r_{V, k}(x) .
$$

Indeed, this is the fact accounting for Theorem 2 above. Thus to show that $\mathbf{R}$ contains the obvious invariants, equation (1) and the fact that rank plus nullity is a constant indicate that we need only show $r_{V, k}$ is in $\mathbf{R}$ for all $L$-modules $V$ and all $k$.

THEOREM 4. Let $L$ be a semisimple Lie algebra and let $N$ be the subvariety of nilpotent elements. Let $\mathbf{R}$ be the ring of integral invariant functions on $N$ generated by $\left\{r_{V, k} \mid V\right.$ a fundamental $L$-module $\}$. Then $r_{V, k} \in \mathbf{R}$ for every $L$-module $V$ and all $k$.

Proof. Let $V_{i}$ stand for the $i$ th fundamental $L$-module. 
Step 1. If $r_{V, k} \in \mathbf{R}$ for some module $V$ and all $k$, then $r_{V \otimes V_{i}, k} \in \mathbf{R}$ for each $i$ and all $k$.

Let $W=V \otimes V_{i}$. Then $x_{W}=x_{V} \otimes 1+1 \otimes x_{V_{i}}$. Let $J(k)$ be the $k \times k$ Jordan block matrix with ones on the subdiagonal and zeros elsewhere. Any nilpotent matrix is similar to a direct sum of matrices $\bigoplus_{k} n_{k} J(k)\left(n_{k} J(k)\right.$ is a direct sum of $n_{k}$ copies of $J(k)$ ) via its Jordan form. Writing $x_{V}$ and $x_{V_{i}}$ in this fashion, and expanding $x_{W}=x_{V} \otimes 1+1 \otimes x_{V}$, one obtains

$$
\left(x_{W}\right)^{t} \sim \bigoplus_{k, j} n_{V, k}(x) n_{V_{i j}}(x)(J(k) \otimes 1+1 \otimes J(j))^{t}
$$

where $\sim$ denotes similarlity. Thus, taking ranks in (2), we arrive at

$$
r_{W, t}(x)=\sum_{k, j} n_{V, k}(x) n_{V_{i j}}(x) \operatorname{rank}(J(k) \otimes 1+1 \otimes J(j))^{t} .
$$

Now by assumption and by equation (1) we have $n_{V, k} \in \mathbf{R}$, and by definition of $\mathbf{R}$ and equation (1) we have $n_{V_{i j}} \in \mathbf{R}$. Furthermore, $\operatorname{rank}(J(k) \otimes 1+1 \otimes$ $J(j))^{t}$ is an integer independent of $x$. Thus $r_{W, t} \in \mathbf{R}$ for all $t$ and this step is complete.

Step 2. If $V$ is a tensor product of $n$ fundamental modules, then all $r_{V, k}$ are in $\mathbf{R}$.

Use Step 1 and induce on $n$.

Step 3. Let $V=V_{\lambda}$ be the irreducible $L$-module of highest weight $\lambda$ on some Cartan subalgebra in which a system of positive roots has been chosen. Then $r_{V, k} \in \mathbf{R}$ for all $k$.

We go by induction on the partial ordering of weights. For weights $\lambda, \gamma$ one has $\gamma<\lambda$ if $\lambda-\gamma$ is a sum of positive roots. Irreducible modules are ordered by the ordering of their highest weights. The minimal modules in this ordering are known to be among the fundamental modules and the trivial module. If $V$ is the trivial $L$-module then all $r_{V, k} \equiv 0$. So by remarks above and the definition of $\mathbf{R}$, we need only consider the induction step. So suppose that $\lambda=\sum n_{i} \lambda_{i}\left(\lambda_{i}\right.$ the $i$ th fundamental weight) is not minimal, and that $r_{V_{r}, k} \in \mathbf{R}$ for all highest weights $\gamma<\lambda$ and all $k$. Set $U=\otimes_{i} \otimes n_{i} V_{\lambda_{i}}$. From the representation theory of $L$,

$$
U=V_{\lambda} \oplus \sum_{\gamma \text { in } A} V_{\gamma}
$$

where $A$ is some set (possibly with repetitions) of highest weights $\gamma<\lambda$. So

$$
r_{U, k}(x)=r_{V_{\lambda}, k}(x)+\sum_{\gamma \in A} r_{V_{\gamma}, k}(x)
$$

By Step 2 the left side of (4) is in $\mathbf{R}$, and the summation in the right side of (4) belongs to $\mathbf{R}$ by the induction assumption. Thus $r_{V_{\lambda}, k} \in \mathbf{R}$ for each irreducible $L$-module $V_{\lambda}$ and all $k$. Now rank is additive across direct sums, and each module is a direct sum of irreducible modules. Thus all $r_{V, k} \in R$. 


\section{REFERENCES}

1. M. Gauger, Conjugacy in a semi-simple Lie algebra is determined by similarity under fundamental representations, J. Algebra (to appear).

2. B. Kostant, Lie group representations on polynomial rings, Amer. J. Math. 85 (1963), 327-404.

3. C. C. MacDuffe, The theory of matrices, Chelsea, New York, 1946.

Department of Mathematics, University of Massachusetts, Amherst, MassachusetTs 01003 